\title{
Oncogenic and incidental HPV types associated with histologically confirmed cervical intraepithelial neoplasia in HIV-positive and HIV-negative South African women
}

\author{
M C van Aardt, ${ }^{1}$ FCOG (SA); G Dreyer, ${ }^{1}$ PhD; L C Snyman, ${ }^{1}$ FCOG (SA); K L Richter, ${ }^{2}$ FCPath (SA); P Becker, ${ }^{3}$ PhD; \\ S M Mojaki, ${ }^{1}$ FCOG (SA) \\ ${ }^{1}$ Gynaecological Oncology Unit, Department of Obstetrics and Gynaecology, School of Medicine, Faculty of Health Sciences, University of \\ Pretoria, South Africa \\ ${ }^{2}$ Department of Medical Virology, School of Medicine, Faculty of Health Sciences, University of Pretoria; National Health Laboratory Service, \\ Pretoria, South Africa \\ ${ }^{3}$ Biostatistics Unit, South African Medical Research Council, Pretoria
}

Corresponding author: M C van Aardt (mc@vanaardt.net)

\begin{abstract}
Background. In Africa, data on the relationship between oncogenic human papillomavirus (HPV) types, immune status and cervical preinvasive lesions are lacking.

Methods. We investigated low-risk (lrHPV) and high-risk (hrHPV) HPV types in a cohort of women with cervical intraepithelial neoplasia (CIN) II/III confirmed on histological examination, in an urban setting with a high prevalence of HIV infection.

Results. Of 270 women with confirmed CIN II/III, 45 were HIV-negative and 225 HIV-positive. HIV-infected women had significantly more HPV type infections, including all HPV $(p<0.001)$ and hrHPV $(p=0.014)$ types. The prevalences of one or more hrHPV type/s were $93.3 \%$ and $92.9 \%$ in HIV-negative and positive patients, respectively. The most prevalent hrHPV type among HIV-negative women was HPV 16, followed by HPV 52, 31, 35 and 58. Among HIV-positive women, HPV 16 was followed by HPV 58, 35, 51 and 52 . Not yet qualifying for highly active antiretroviral therapy (HAART) (CD4 count $>350$ cells $/ \mu \mathrm{L}$ ) or having received HAART for $\geq 12$ months were negatively associated with HPV 18, 33, 45, 51, 52, 59 and 82.

Conclusions. In South Africa, burdened by the HIV pandemic, high numbers of high- and low-risk HPV type infections are present in women with cervical preneoplasia. HPV type distribution differs among varying levels of HIV-induced immune depletion.

S Afr Med J 2016;106(6):617-622. DOI:10.7196/SAMJ.2016.v106i6.10335
\end{abstract}

Cervical cytological examination is limited to inspection of cells, and the diagnosis of cervical intraepithelial neoplasia (CIN) requires the examination of cervical tissue to make a histological diagnosis. The risk of progression to cervical cancer is greatest for women with CIN III..$^{[1]}$

One of the known predisposing causes for these preneoplastic changes is persistent infection with one or more of the human papillomavirus (HPV) types. The immune system's inability to resist changes plays a vital role in the development of cervical carcinoma. ${ }^{[2]}$ Immunocompromised individuals have an increased risk of cervical neoplasia. ${ }^{[3,4]}$

Globally, with an estimated 33 million people infected, the HIV/ AIDS pandemic is placing a huge burden on healthcare systems and has an enormous impact on women of all ages. ${ }^{[5,6]}$ Approximately $75 \%$ of women infected with HIV are living in sub-Saharan Africa, and South Africa (SA) has more HIV-infected women than any other country. ${ }^{[7,8]}$

It is well established that HIV-infected women have an increased susceptibility to HPV infections and HPV-associated lesions, which include CIN II/III and cervical cancer. ${ }^{[7,9-13]}$ High-risk HPV (hrHPV) types and CIN are up to four times more common in HIV-positive women. ${ }^{[14]}$ Even though HPV 16 and 18 are responsible for up to $70 \%$ of cervical cancers, high-grade cervical lesions are more likely to be associated with non-HPV 16/18 types, especially among HIVinfected women. ${ }^{[15,16]}$

In women with HIV coinfection, studies have shown that the chance of finding cervical HPV DNA together with abnormal cervical cytology increases as the CD4 cell count decreases. ${ }^{[17]} \mathrm{A}$ decline in CD4 cell count and rising HIV viral load are both risk factors for invasive cervical lesions. ${ }^{[6]}$ However, some aspects of the relationship between CIN, immune depletion and the effect of highly active antiretroviral therapy (HAART) are not yet clear. ${ }^{[5]}$

Some data suggest that despite initiation of HAART and the associated CD4 cell count increase, most women will not experience regression of high-grade (CIN II/III) lesions. ${ }^{[18,19]}$ It appears that immune status has a minimal role in either regression of high-grade lesions or cervical cancer advancing from these lesions. Although immune depletion results in an increased chance of premalignant cervical disease, it seems that other factors contribute to the development of cervical cancer from high-grade cervical lesions. ${ }^{[18,19]}$

Local data from Africa on the relationship between oncogenic HPV types, immune status and cervical preinvasive lesions are also incomplete. ${ }^{[20,21]}$ The majority of studies reported in the literature used cytology results synonymously with cervical premalignant changes. ${ }^{[6,22]}$ As cytology is only a screening test, it is important to compare the prevalence of oncogenic HPV types with histopathologically confirmed CIN in women with different levels of immune competence.

\section{Methods \\ Study design}

This was a descriptive study performed at the Gynaecological Oncology Unit at Steve Biko Academic Hospital and the University of Pretoria, SA. Data were obtained from 1 July 2010 to 30 August 2013. 
Patients included in the study were women aged $\geq 18$ years, referred for treatment of high-grade squamous intraepithelial lesions (HSILs) detected on conventional cervical cytological examination (Papanicolaou smears) as part of the national screening programme. The study population was representative of women attending public healthcare facilities in the Tshwane region.

\section{Consent process and ethical considerations}

Patients received counselling and an information document that explained the method and voluntary nature of the study. During counselling by trained nursing personnel, patients were motivated to undergo HIV testing as per standard departmental management protocols. It was clearly explained to them that testing was voluntary and not a prerequisite for treatment. Planned treatment was also explained: large loop excision of the transformation zone (LLETZ), or directed biopsies if malignancy was suspected. All patients were informed of their HIV results, if applicable. All patients tested for HIV received post-test counselling and were offered a CD4 cell count. These patients were referred to the appropriate antiretroviral therapy clinic for further management. This study was approved by the Research Ethics Committee of the Faculty of Health Sciences of the University of Pretoria (Refs 26/2010, 189/2012).

\section{Patient recruitment}

Three hundred and thirty-four consecutive patients referred with HSILs detected on cervical cytological examination were invited to participate in the study. Histological results were available for all patients. Only the 270 patients with confirmed CIN II or III were included in the final study analysis. Of the 64 patients excluded, 10 had cervical cancer, 25 had CIN I, 12 had cervicitis and 8 had no histological abnormalities. The specimens were inadequate for histological examination in 9 cases. The CD4 cell count was recorded, if applicable, as was treatment with HAART. Based on the 2010 SA guidelines, ${ }^{[23]}$ HAART was divided into different groups: patients not yet qualifying for HAART treatment (CD4 count $>350$ cells $/ \mu \mathrm{L})$; patients in the process of initiating HAART (CD4 count $\leq 350$ cells/ $\mu \mathrm{L}$ ); and patients treated with HAART for $<6$ months, 6 - 12 months and $>12$ months.

\section{Sample collection and transport}

Patients underwent colposcopic evaluation and a LLETZ procedure or biopsy as indicated. Samples were placed in buffered formalin and transported to the Department of Anatomical Pathology at the University of Pretoria, where histological examination was performed.

HPV testing, using a dry swab, was performed on all participating patients. After collection, the swabs were transported in phosphate-buffered saline and $10 \%$ methanol solution to the Department of Medical Virology at the University of Pretoria, where HPV DNA testing was performed.

\section{HPV DNA testing}

DNA extraction was accomplished by means of the DNA Isolation Kit (Roche Molecular Systems, USA) on the MagNa Pure automated extraction system (Roche, USA). An HPV linear array genotyping kit (Roche Molecular Systems) was used to determine the HPV type. Fifteen high-risk types (HPV 16, 18, 31, 33, 35, 39, 45, 51, 52, $56,58,59,68,73$ and 82 ), three probable high-risk types (HPV 26, 53 and 66) and 19 low/undetermined risk types (HPV 6, $11,40,42,54,55,61,62,64,67,69,70,71$, $72,81,83,84$, IS39 and CP6108) were tested for. ${ }^{[24}$

\section{Data capturing and analysis}

Data were captured on Excel datasheets (2011, Microsoft, USA), and analysis was performed using Stata statistical software release 11 (StataCorp, USA). Discrete data were mainly binary in nature and summary statistics were frequency, percentage, 95\% confidence intervals (CIs), cross-tables and bar charts. Continuous data were summarised using descriptive statistics, means and standard deviations (SDs) along with 95\% CIs. Comparison between groups was done with Fisher's exact test for discrete outcomes and Student's two-sample $t$-test or the Wilcoxon rank sum test for continuous outcomes. Testing was done at the 0.05 level of significance. Furthermore, the association between high-risk virus type and HAART use was assessed using logistic regression analysis and adjusted for age and CD4 cell count (logarithmic scale).

\section{Results}

The ages of the patients ranged from 21 to 66 years. HIV results were available for all women. Of the 270 women, 225 (83.3\%) were HIV-infected and 45 (16.7\%) were not infected. There was no significant difference between the two groups with regard to age of diagnosis $(p=0.186)$. CD4 cell counts were available for 205 of the 225 HIVinfected patients (91.1\%), and information on HAART treatment and duration was available for all (Fig. 1).

\section{HPV prevalence}

The prevalence of any HPV type in patients with CIN II/III was $96.7 \%$, $97.8 \%$ among HIV-negative patients and $96.4 \%$ among HIV-positive patients. The prevalence of one or more hrHPV type/s was $93.0 \%$ in the entire population and $93.3 \%$ and $92.9 \%$ in HIV-negative and HIV-positive patients, respectively. Twenty-seven HIV-negative patients (60.0\%) and 42 HIV-positive patients (18.7\%) did not have any low-risk HPV (lrHPV) DNA detected.

\section{Single and multiple hrHPV type infections}

A total of 119 HPVs were detected in the 45 HIV-non-infected patients, of which 82 were hrHPV. The total number of HPV types detected in the 225 HIV-infected women was 1090 , with 577 of these categorised as hrHPV types. The number of HPV types detected per patient was significantly greater among HIV-infected v. non-infected patients for all HPV types $(p<0.001)$ and for hrHPV types $(p=0.014)$ (Fig. 1$)$.

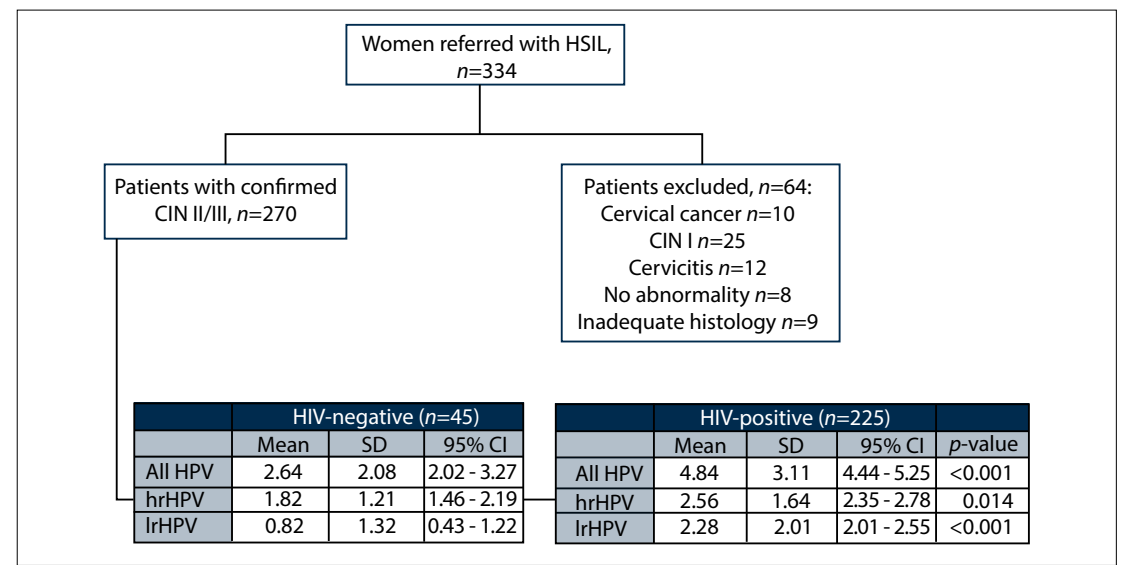

Fig. 1. Descriptive statistics at baseline of the study population and average number of HPV infections per patient. 
One-third $(33.3 \%)$ of HIV-negative patients had a single HPV type infection, as opposed to only $18 \mathrm{HIV}$-positive patients $(8.0 \%)$. Multiple HPV types were detected in $29 \mathrm{HIV}$-negative patients (64.4\%) and $183 \mathrm{HIV}$-positive patients (81.3\%). Three HIV-negative patients (6.7\%) had no hrHPV infections, 20 (44.4\%) were infected with a single hrHPV type, and the remaining patients $(48.9 \%)$ had two or more hrHPV type infections. Forty-four patients (19.6\%) coinfected with HIV had a single hrHPV type detected and 165 (73.3\%) had multiple hrHPV type infections.

\section{HPV type distribution}

In the group as a whole, the most prevalent hrHPV types, in descending order of frequency, were HPV 16, 58, 35, 52, 51 and 45. The most prevalent hrHPV type in the HIV-negative group was HPV 16 (Fig. 2), followed by HPV 52, 31, 35, 58, 18, 33 and 45. HPV 84 was the most prevalent lrHPV type. In the HIV-infected group, HPV 16 was also the most prevalent hrHPV type, followed by HPV 58, 35, 51, 52, 45, 18 and 31 . The most prevalent lrHPV type was HPV 62.

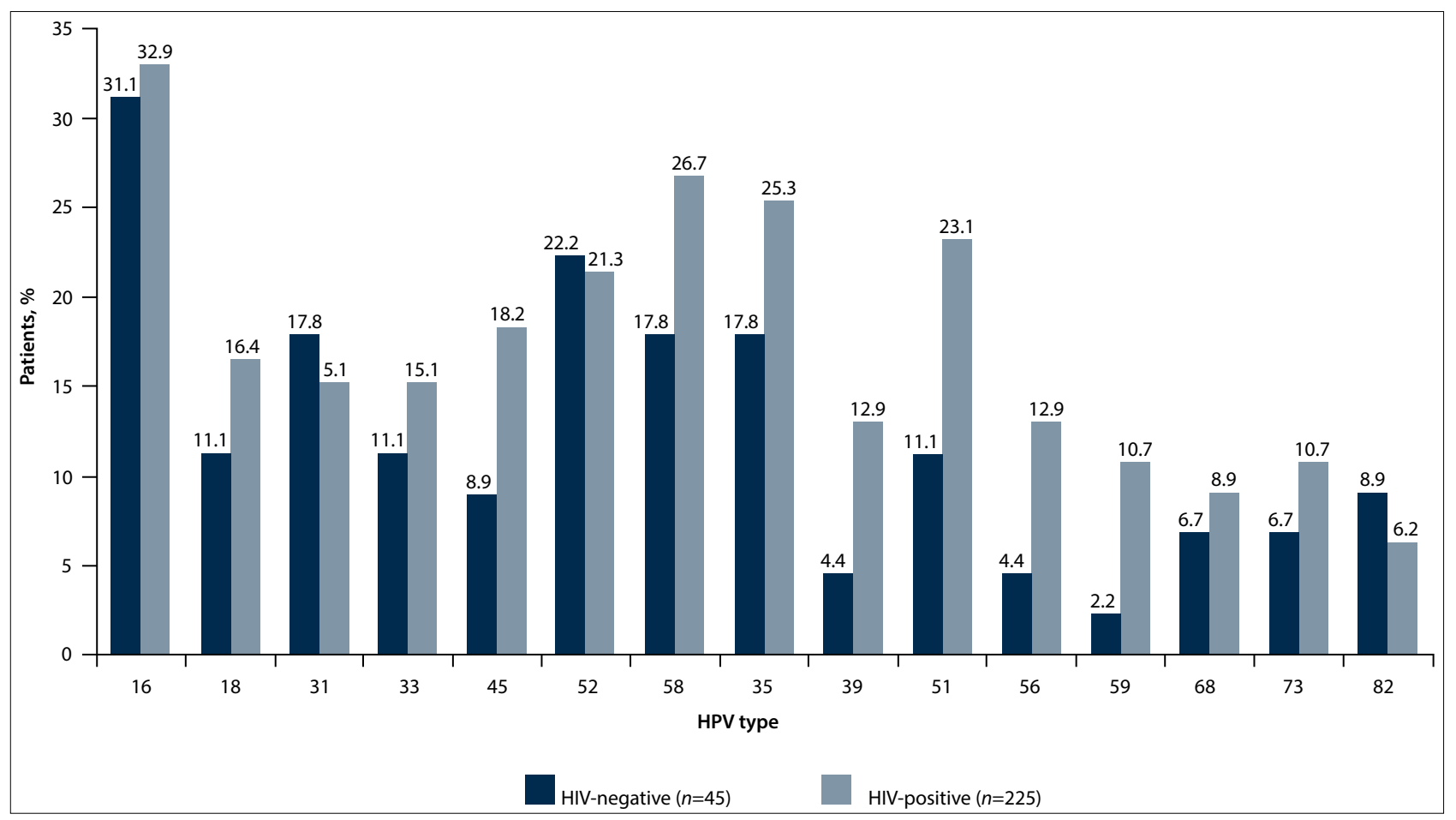

Fig. 2. Distribution of hrHPV types among HIV-negative and positive patients with CIN II/III.

Table 1. hrHPV type distribution in relation to HAART treatment

\begin{tabular}{|c|c|c|c|c|c|c|c|c|}
\hline $\begin{array}{l}\text { HPV } \\
\text { type }\end{array}$ & $\begin{array}{l}\text { HIV- } \\
(N=45), \\
n(\%)\end{array}$ & $\begin{array}{l}\text { No ARV } \\
(N=35), \\
n(\%)\end{array}$ & $\begin{array}{l}\text { ARV > } 12 \text { mo } \\
(N=43), \\
n(\%)\end{array}$ & $\begin{array}{l}\text { ARV } 6-12 \\
\text { mo }(N=37), \\
n(\%)\end{array}$ & $\begin{array}{l}\text { ARV <6 mo } \\
(N=56) \\
n(\%)\end{array}$ & $\begin{array}{l}\text { Start ARV } \\
(N=54), \\
n(\%)\end{array}$ & $\begin{array}{l}\text { Total } \\
(N=270), \\
n(\%)\end{array}$ & $p$-value \\
\hline 16 & $14(31.1)$ & $15(42.9)$ & $11(25.6)$ & 7 (18.9) & $22(39.3)$ & $19(35.2)$ & $88(32.6)$ & 0.206 \\
\hline 18 & $5(11.1)$ & $5(14.3)$ & $7(16.3)$ & $3(8.1)$ & $11(19.6)$ & $11(20.4)$ & $42(15.6)$ & 0.570 \\
\hline 31 & $8(17.8)$ & $5(14.3)$ & $3(7.0)$ & 7 (18.9) & $11(19.6)$ & $8(14.8)$ & $42(15.6)$ & 0.562 \\
\hline 33 & $5(11.1)$ & $4(11.4)$ & $10(23.3)$ & 7 (18.9) & $6(10.7)$ & $7(13.0)$ & $39(14.4)$ & 0.494 \\
\hline 35 & $8(17.8)$ & $5(14.3)$ & $10(23.3)$ & $12(32.4)$ & $15(26.8)$ & $15(27.8)$ & $65(24.1)$ & 0.434 \\
\hline 39 & $2(4.4)$ & $3(8.6)$ & $4(9.3)$ & 7 (18.9) & $8(14.3)$ & $7(13.0)$ & $31(11.5)$ & 0.384 \\
\hline 45 & $4(8.9)$ & $5(14.3)$ & 9 (20.9) & $6(16.2)$ & $11(19.6)$ & $10(18.5)$ & $45(16.7)$ & 0.659 \\
\hline 51 & $5(11.1)$ & $7(20.0)$ & 8 (18.6) & 8 (21.6) & $15(26.8)$ & $14(25.9)$ & $57(21.1)$ & 0.433 \\
\hline 52 & $10(22.2)$ & 9 (25.7) & 9 (20.9) & $11(29.7)$ & $12(21.4)$ & $7(13.0)$ & $58(21.5)$ & 0.492 \\
\hline 56 & $2(4.4)$ & $2(5.7)$ & $6(13.9)$ & $5(13.5)$ & $8(14.3)$ & $8(14.8)$ & $31(11.5)$ & 0.412 \\
\hline 58 & $8(17.8)$ & 8 (22.9) & $8(18.6)$ & $16(43.2)$ & $15(26.8)$ & $13(24.1)$ & $68(25.2)$ & 0.141 \\
\hline 59 & $1(2.2)$ & $5(14.3)$ & $2(4.6)$ & 7 (18.9) & $4(7.1)$ & $6(11.1)$ & $25(9.3)$ & 0.092 \\
\hline 68 & $3(6.7)$ & $2(5.7)$ & $3(7.0)$ & $4(10.8)$ & $4(7.1)$ & $7(13.0)$ & $23(8.5)$ & 0.826 \\
\hline 73 & $3(6.7)$ & $1(2.9)$ & $2(4.6)$ & $4(10.8)$ & $11(19.6)$ & $6(11.3)$ & $27(10.0)$ & 0.111 \\
\hline 82 & $4(8.9)$ & $2(5.7)$ & 5 (11.6) & $2(5.4)$ & $1(1.8)$ & $4(7.4)$ & $18(6.7)$ & 0.451 \\
\hline
\end{tabular}




\section{Association of hrHPV types with HAART use}

The prevalence of hrHPV infections was lowest among patients who had been receiving HAART for $>12$ months. Except for patients who had been receiving HAART for between 6 and 12 months, HPV 16 was the most prevalent hrHPV type among all the different subgroups. Table 1 illustrates the distribution of the different hrHPV types in relation to HAART use.

Patients were divided into two groups. Firstly, patients not yet qualifying for HAART (CD4 count $>350$ cells $/ \mu \mathrm{L}$ ) and those who had been receiving HAART for $\geq 12$ months were grouped together. This group was compared with a second group comprising patients in the process of initiating HAART (CD4 count $\leq 350$ cells/ $\mu \mathrm{L})$ and patients who had been receiving HAART for $<12$ months. Adjusted for age and CD4 cell count on a logarithmic scale, the odds of being infected with HPV 18, 33, $45,51,52,59$ and 82 were lower in patients not yet requiring HAART or on HAART for longer than 12 months (Table 2). There was a significant difference for HPV 33 $(p=0.029), 59(p=0.009)$ and $82(p=0.034)$ infections. The odds of having an HPV 73 $(p=0.004)$ infection were significantly lower in patients who had been receiving HAART for $<12$ months or were in the process of initiating HAART.

\section{Vaccine-preventable infections}

We investigated: (i) the prevalence of HPV 16 and/or 18 infections alone, without any other hrHPV coinfections; (ii) only the rates of infection with the seven hrHPV types (HPV 16, 18, 31, 33, 45, 52 and 58) covered by the nine-valent vaccine; and (iii) rates of infection with other hrHPV types currently not specifically covered by vaccines.

As illustrated in Fig. 3, HPV 16 and/or 18 were detected in 124 patients $(45.9 \%)$ and $214(79.3 \%)$ were infected with HPV 16, $18,31,33,52$ and/or 58. Only 37 patients (7.1\%) were infected with hrHPV types not included in the nine-valent vaccine. The distribution of hrHPV types between HIVinfected and non-infected patients was very similar.

\section{Discussion \\ Background}

The distribution of HPV types in this cohort of SA women with histologically confirmed CIN II/III is very important. In this study, the positive predictive value for CIN II or more severe changes for patients referred with cytological evidence of HSILs was $83.6 \%$. This highlights the importance of

\begin{tabular}{|c|c|c|c|c|}
\hline HPV type & Crude OR & Adjusted $\mathbf{O R}^{\dagger}$ & $95 \% \mathrm{CI}$ & $p$-value \\
\hline 16 & 1.02 & 1.17 & $0.58-2.37$ & 0.120 \\
\hline 18 & 1.23 & 0.84 & $0.34-2.09$ & 0.282 \\
\hline 31 & 2.15 & 2.08 & $0.77-5.59$ & 0.169 \\
\hline 33 & 0.67 & 0.54 & $0.21-1.36$ & $0.029^{*}$ \\
\hline 35 & 1.44 & 1.23 & $0.57-2.65$ & 0.551 \\
\hline 39 & 1.75 & 1.87 & $0.68-5.12$ & 0.639 \\
\hline 45 & 0.95 & 0.78 & $0.34-1.83$ & 0.685 \\
\hline 51 & 1.35 & 0.93 & $0.42-2.05$ & 0.164 \\
\hline 52 & 0.66 & 0.98 & $0.43-2.22$ & 0.132 \\
\hline 56 & 1.42 & 1.24 & $0.47-3.28$ & 0.739 \\
\hline 58 & 1.51 & 1.43 & $0.65-3.17$ & 0.146 \\
\hline 59 & 1.13 & 0.93 & $0.30-2.84$ & $0.009^{*}$ \\
\hline 68 & 1.39 & 1.50 & $0.45-5.01$ & 0.904 \\
\hline 73 & 4.15 & 2.39 & $0.64-8.96$ & $0.004^{*}$ \\
\hline 82 & 0.49 & 0.23 & $0.06-0.86$ & $0.034^{*}$ \\
\hline \multicolumn{5}{|c|}{$\begin{array}{l}\mathrm{OR}=\text { odds ratio. } \\
{ }^{*} \text { Statistically significant. }\end{array}$} \\
\hline
\end{tabular}

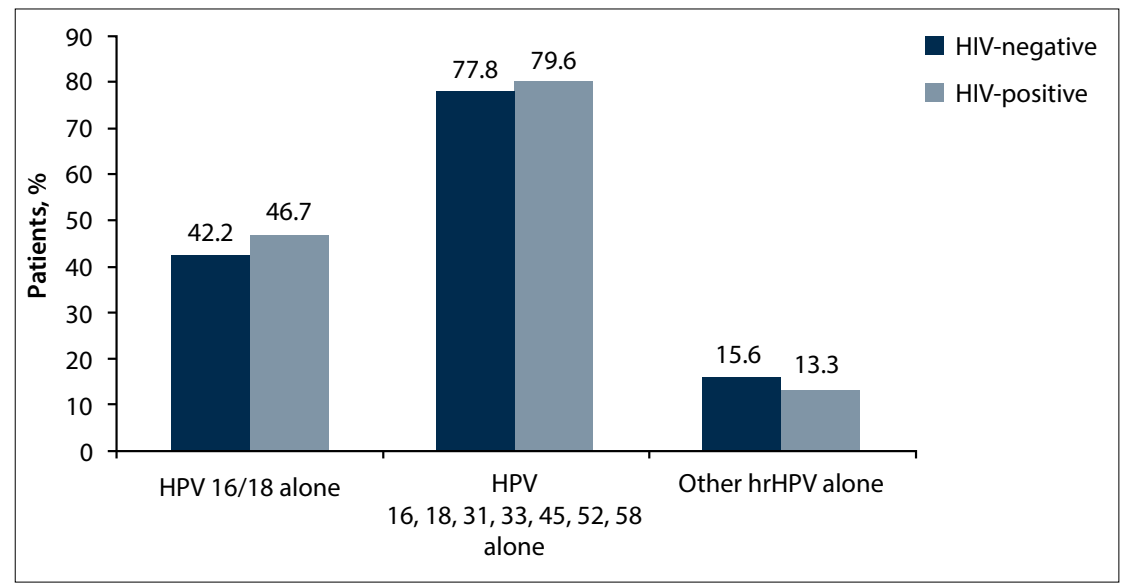

Fig. 3. hrHPV type distribution of vaccine-preventable infections.

using histopathologically confirmed cervical lesions as the endpoint in the study of cervical disease. ${ }^{[25]}$

More than $80 \%$ of patients in this study were HIV-infected. Although not an AIDSdefining disease like invasive cervical cancer, CIN is regarded as an HIV-related disease. ${ }^{[5]}$ The HIV status of all patients was known, reflecting positively on a high uptake of voluntary HIV testing after appropriate counselling. Although premalignant lesions are more prevalent in HIV-infected women, because they come into contact with the health system relatively often, it is likely that they are better screened than women in the general population, which may explain the high rate of HIV infection in this study. The large percentage of patients infected with
HIV also highlights the burden that the HIV/ AIDS pandemic places on SA's healthcare system. Along with the risk of CIN among HIV-infected women, the risk of genital HIV shedding is significantly elevated in the presence of CIN, leading to an increased possibility of HIV transmission. ${ }^{[5]}$

\section{HPV prevalence}

The prevalence of any HPV in the current study was $96.7 \%$. A recent meta-analysis reported the global prevalence of all HPV types in women with CIN II/III as ranging from $86 \%$ to $93 \%{ }^{[26]}$ In 2012 , the prevalence in Africa was reported as $89 \%$ for CIN II and 83\% for CIN III. ${ }^{[26]}$

The prevalence of one or more hrHPV types $(93 \%)$ was similar to a Botswana study 
(92\%), but higher than prevalences reported from Kenya (82\%) and SA (75\%). ${ }^{[7,5,27]}$ A study from Spain found an hrHPV prevalence of $100 \%$ among 18 HIV-positive patients presenting with cytological evidence of HSILs. ${ }^{[10]}$

The prevalence of hrHPV was higher among patients with a CD4 cell count $<200$ cells $/ \mu \mathrm{L}(97.3 \%)$ than among patients with a CD4 cell count of $\geq 200$ cells $/ \mu \mathrm{L}$ (90.1\%). This is considerably higher than the overall prevalence $(84.1 \%)$ of HPV infections in HIV-positive women with HSILs reported by Clifford et al. ${ }^{[28]}$

\section{Single and multiple HPV type infections}

Compared with the HIV-negative cohort of patients in the study by McDonald et al., ${ }^{[27]}$ HIV-negative patients in this study had more multiple hrHPV type infections (49\% v. 20\%). Women infected with HIV are often coinfected with multiple types, as well as with a broader spectrum of HPV types. ${ }^{[16,29,30]}$ There were more infections with multiple HPV types in this study than reported by Guan et al. ${ }^{[26]}$ The 73.3\% of HIV-positive patients coinfected with multiple hrHPV types represents a much higher proportion than reported in other studies $(27.8-56 \%){ }^{[7,15,22]}$

\section{Specific HPV types}

In both the HIV-negative and HIV-positive groups, HPV 16 was the most prevalent hrHPV type, with almost one-third of patients infected. Disregarding HIV status, the most common hrHPV types identified were, in decreasing order of prevalence, HPV 16, 58, $35,52,51,45$ and 31 . This distribution differed from the metaanalysis, compared with both global and African data, on HPV type distribution in patients with cytological evidence of HSILs published in 2006. ${ }^{[31]}$ The most common types of hrHPV among patients with CIN II or CIN III, as illustrated by a more recent meta-analysis, were HPV 16 followed by HPV 52, 58, 31, 18, 33, 45 and 35. ${ }^{[26]}$ There has been a worldwide increase in the prevalence of HPV 52 and 58 over the past 10 years. ${ }^{[15]}$ Chen et al..$^{[32]}$ suggested that the long-term risk for developing cervical cancer was higher for HPV 58 than other non-HPV 16 types.

The just under $33 \%$ prevalence of HPV 16 in the entire study population is comparable to the prevalence reported by Guan et al. ${ }^{[26]}$ for Africa (30.3\%), which included both patients with CIN II/III and HSILs. The specific regions of Africa were not specified. The prevalence of just under $33 \%$ is also comparable to the global prevalence (34.7 $52 \%)$ reported by Clifford et al. ${ }^{[31]}$ for patients with HSILs. However, the prevalence of HPV 16 (32.9\%) among HIV-infected patients in this study is lower than in other reports on HIV-positive patients from Europe, SA and Botswana (37.5 - 45\%), but also higher than reported in other African studies $(26.5-29.4 \%)^{[7,10,15,21,30,33]}$

\section{HPV and HAART use}

Findings from a large review on the impact of HAART on HPV and CIN did not show a significant difference for women on HAART in relation to HPV and disease clearance. This study was limited by short follow-up periods of up to 24 months and did not account for treatment compliance. ${ }^{[34]}$ More recent studies, however, have suggested that HrHPV infections can be cleared after a lengthy period of optimal HIV control and restoration of immune function. ${ }^{[35-38]}$ The lower prevalence of HPV 16 among women treated with HAART for $>6$ months in this study may demonstrate a protective effect against new infections or improve clearance.

\section{Study limitations}

Limitations of the study include the fact that the patients who took part were from the referral areas served by Steve Biko Academic
Hospital, so the findings cannot be extrapolated to the rest of SA. The small number of HIV-non-infected patients included is also a limitation. Although important information on hrHPV distribution among patients on HAART was obtained, little could be concluded regarding the effect of HAART-induced immune reconstitution on cervical lesion regression and persistent infections. A longitudinal study will be needed to evaluate this. This study also reported only on HPV DNA detected on the cervical surface and highlights the need to determine the specific HPV type or types incorporated within the specific lesion. Our unit is currently busy with a study comparing HPV types obtained from CIN lesions with surface HPV types.

\section{Conclusion}

In SA, burdened by the HIV pandemic, high numbers of high- and low-risk HPV type infections are present in women with cervical preneoplasia. The distribution of HPV types differs in HIV-infected patients. Administering the nine-valent HPV vaccine to women in our population may prevent as many as $80 \%$ of CIN II/III lesions. Not yet requiring HAART or having been receiving HAART for $>12$ months appear to be negatively associated with HPV 33, 59 and 82, and positively associated with HPV 73 . Knowledge about the specific HPV type distribution is crucial to direct development of future HPV vaccines and to guide HPV-based screening in both HIVinfected and non-infected patients in this population.

\section{References}

1. Hoppenot C, Stampler K, Dunton C. Cervical cancer screening in high- and low-resource countries Implications and new developments. Obstet Gynecol Surv 2012;67(10):658-667. DOI:10.1097 OGX.0b013e3182732375

2. Joshi SN, Gopalkrishna V, Kumar BK, et al. Cervical squamous intra-epithelial changes and human papillomavirus infection in women infected with human immunodeficiency virus in Pune, India. Med Virol 2005;76(4):470-475. DOI:10.1002/jmv.20385

3. Tweddel G, Heller P, Cunnane M, et al. The correlation between HIV seropositivity, cervical dysplasia, and HPV subtypes 6/11, 16/18,31/33/35. Gynecol Oncol 1994;52(2):161-164. DOI:10.1006/gyno.1994.1024

Gadducci A, Barsotti C, Cosio S, et al. Smoking habit, immune suppression, oral contraceptive use, and hormone replacement therapy use and cervical carcinogenesis: A review of the literature. Gynecol Endocrinol 2011;27(8):597-604. DOI:10.3109/09513590.2011.558953

5. Pantanowitz L, Michelow P. Review of human immunodeficiency virus (HIV) and squamous lesions of the . Pantanowitz L, Michelow P. Review of human immunodeficiency virus (HIV)
uterine cervix. Diagn Cytopathol 2011;39(1):65-72. DOI:10.1002/dc.21364

uterine cervix. Diagn Cytopathol 2011;39(1):65-72. DOI:10.1002/dc.21364
6. Teixeira NC, Araújo AC, Correa CM, et al. Prevalence and risk factors for cervical intraepithelial neoplasia 6. Teixeira NC, Araújo AC, Correa CM, et al. Prevalence and risk factors for cervical intraepithelial neoplasia
among HIV-infected women. Braz J Infect Dis 2012;16(2):164-169. DOI:10.1590/S1413-86702012000200010 among HIV-infected women. Braz Infect Dis 2012;16(2):164-169. DOI:10.1590/S1413-86702012000200010
7. De Vuyst H, Mugo NR, Chung MH, et al. Prevalence and determinants of human papillomavirus infection and cervical lesions in HIV-positive women in Kenya. Br J Cancer 2012;107(9):1624-1630. DOI:10.1038/bjc.2012.441

8. Adler DH, Kakinami L, Modisenyane T, et al. Increased regression and decreased incidence of human papillomavirus-related cervical lesions among HIV-infected women on HAART. AIDS 2012;26(13):1645-1652. DOI:10.1097/QAD.0b013e32835536a3

9. Joshi S, Sankaranarayanan R, Muwonge R, et al. Screening of cervical neoplasia in HIV-infected women in India. AIDS 2013;27(4):607-615. DOI:10.1097/QAD.0b013e32835b1041

10. Stuardo V, Agustí C, Godinez JM, et al. Human papillomavirus infection in HIV-1 infected women in Catalonia (Spain): implications for prevention of cervical cancer. PLoS One 2012;7(10):e47755. DOI:10.1371/journal.pone.0047755

11. Zimmermmann JB, Gobbi H, Alves MJ, et al. Langerhans cell density in cervical intraepithelial neoplasia associated with human papillomavirus infection in HIV-infected and HIV-noninfected neoplasia associated with human papillomavirus infection in HIV-infected and HIV-noninfected
Brazilian women. Int J Gynecol Cancer 2012;22(8):1291-1296. DOI:10.1097/IGC.0b013e318263ef88

Brazilian women. Int I Gynecol Cancer 2012;22(8):1291-1296. DOI:10.1097/IGC.0b013e318263ef88
12. Massad LS, Xie X, Darragh TM, et al. Histologic correlates of glandular abnormalities in cervical

Massad LS, Xie X, Darragh TM, et al. Histologic correlates of glandular abnormalities in cervical
cytology among women with human immunodeficiency virus. Obstet Gynecol 2009;114(5):1063cytology among women with human immun
1068. DOI:10.1097/AOG.0b013e3181bc6ce0

13. Massad LS, Evans CT, Weber KM, et al. Changes in knowledge of cervical cancer prevention and human papillomavirus among women with human immunodeficiency virus. Obstet Gynecol 2010;116(4):941-947. DOI:10.1097/AOG.0b013e3181f2dbae

14. Peedicayil A, Thiyagarajan $K$, Gnanamony $M$, et al. Prevalence and risk factors for human papillomavirus and cervical intraepithelial neoplasia among HIV-positive women at a tertiary leve hospital in India. J Low Genit Tract Dis 2009;13(3):159-164. DOI:10.1097/LGT.0b013e31818fb40d

15. Ramogola-Masire D, McGrath CM, Barnhart KT, et al. Subtype distribution of human papillomaviru in HIV-infected women with cervical intraepithelial neoplasia stages 2 and 3 in Botswana. Int J Gynecol Pathol 2011;30(6):591-596. DOI:10.1097/PGP.0b013e31821bf2a6

16. Franceschi S, Ronco G. The prevention of cervical cancer in HIV-infected women. AIDS 2010;24(16):2579-2580. DOI:10.1097/QAD.0b013e32833f39b2

17. Burger EA, Kornør H, Klemp M, et al. HPV mRNA tests for the detection of cervical intraepithelial Burger EA, Kornør H, Klemp M, et al. HPV mRNA tests for the detection of cervical intraepithelial
neoplasia: A systematic review. Gynecol Oncol 2011;120(3):430-438. DOI:10.1016/.ygyno.2010.11.013 8. Palefsky JM, Holly EA. Chapter 6: Immunosuppression and Co-infection with HIV. J Natl Cancer Inst Monogr 2003;31:41-46.

Monogr 2003;31:41-46.
19. ACOG Committee on Practice Bulletins - Gynecology. ACOG Practice Bulletin No. 117: Gynecologic care for women with human immunodeficiency virus. Obstet Gynecol 2010;116(6):1492-1509. DOI:10.1097/AOG.0b013e3182054cae

20. Luque AE, Hitti J, Mwachari C, et al. Prevalence of human papillomavirus genotypes in HIV-1-infected women in Seattle, USA and Nairobi, Kenya: Results from the women's HIV interdisciplinary network (WHIN). Int J Infect Dis 2010;14(9):e810-e814. DOI:10.1016/j.ijid.2010.03.016

21. Dols JA, Reid G, Brown JM, et al. HPV type distribution and cervical cytology among HIVpositive Tanzanian and South African women. ISRN Obstet Gynecol 2012;2012:514146. DOI:10.5402/2012/514146 
22. Mane A, Nirmalkar A, Risbud AR, et al. HPV genotype distribution in cervical intraepithelial neoplasia among HIV-infected women in Pune, India. PLoS One 2012;7(6):e38731. DOI:10.1371/ journal.pone.003873

23. National Department of Health, South Africa. The South African Antiretroviral Treatment Guidelines. Pretoria: NDoH, 2010. http://www.uj.ac.za/EN/CorporateServices/ioha/Documentation/Documents/ ART\%20Guideline.pdf (accessed 14 June 2015).

24. Muñoz N, Bosch FX, de Sanjosé S, et al. Epidemiologic classification of human papillomavirus type associated with cervical cancer. N Engl J Med 2003;348(6):518-527. DOI:10.1056/NEJMoa021641

25. Kuhn L, Wang C, Tsai WY, et al. Efficacy of human papillomavirus-based screen-and-treat for cervical cancer prevention among HIV-infected women. AIDS 2010;24(16):2553-2561. DOI:10.1097/ QAD.0b013e32833el63e

26. Guan P, Howell-Jones R, Li N, et al. Human papillomavirus types in 115,789 HPV-positive women: A meta-analysis from cervical infection to cancer. Int I Cancer 2012;131(10):2349-2359. DOI:10.1002/ ijc. 27485

27. McDonald AC, Denny L, Wang C, et al. Distribution of high-risk human papillomavirus genotype among HIV-negative women with and without cervical intraepithelial neoplasia in South Africa. PLoS One 2012:7(9):e44332. DOI:10.1371/journal.pone.0044332

28. Clifford GM, Gonçalves MA, Franceschi S, HPV and HIV Study Group. Human papillomavirus type among women infected with HIV: A meta-analysis. AIDS 2006;20(18):2337-2344. DOI:10.1016/j. ygyno.2009.10.058

29. Peedicayil A, Thiyagarajan K, Gnanamony M, et al. Prevalence and risk factors for human papillomavirus and cervical intraepithelial neoplasia among HIV-positive women at a tertiary leve hospital in India. J Low Genit Tract Dis 2009;13(3):159-164. DOI:10.1097/LGT.0b013e31818fb40d

30. Marais DJ, Passmore JA, Denny L, et al. Cervical and oral human papillomavirus types in HIV-1 positive and negative women with cervical disease in South Africa. J Med Virol 2008;80(6):953-959. DOI:10.1002/jmv.21166
31. Clifford G, Franceschi S, Diaz M, et al. Chapter 3: HPV type-distribution in women with and without cervical neoplastic diseases. Vaccine 2006;24(Suppl 3):S26-S34. DOI:10.1016/j.vaccine.2006.05.026

32. Chen HC, Schiffman M, Lin CY, et al. Persistence of type-specific human papillomavirus infection and increased long-term risk of cervical cancer. J Natl Cancer Inst 2011;103(18):1387-1396. DOI:10.1093/ inci/djr283

33. Heard I, Cubie HA, Mesher D, et al. Characteristics of HPV infection over time in European women who are HIV-1 positive. Br J Obstet Gynaecol 2013;120(1):41-49. DOI:10.1111/1471-0528.12015

34. Bratcher LF, Sahasrabuddhe VV. The impact of antiretroviral therapy on HPV and cervical intraepithelial neoplasia: Current evidence and directions for future research. Infect Agent Cancer 2010;5:8. DOI:10.1186/1750-9378-5-8

35. Fife K, Wu J, Squires K, et al. Prevalence and persistence of cervical human papillomavirus infection in HIV-positive women initiating highly active antiretroviral therapy. J Acquir Immune Defic Syndr 2009;51(3):274-282. DOI:10.1097/QAI.0b013e3181a97be5

36. Minkoff $\mathrm{H}$, Zhong $\mathrm{Y}$, Burk R, et al. Influence of adherent and effective antiretroviral therapy use on human papillomavirus infection and squamous intraepithelial lesions in human immunodeficiency virus-positive women. J Infect Dis 2010;201(5):681-690. DOI:10.1086/650467

37. Konopnicki D, Manigart Y, Gilles C, et al. Sustained viral suppression and higher CD4+ T-cell count reduces the risk of persistent cervical high-risk human papillomavirus infection in HIV-positive reduces the risk of persistent cervical high-risk human papillomavin
women. J Infect Dis 2013;207(11):1723-1729. DOI:10.1093/infdis/ito90

38. Blitz S, Baxter I, Raboud J, et al., Canadian Women's HIV Study Group. Evaluation of HIV and HAART on the natural history of HPV infection and cervical cytopathology in HIV-positive and high risk HIVnegative women. J Infect Dis 2013;208(3):454-462. DOI:10.1093/infdis/jit18

Accepted 23 November 2015 